\title{
The Effects of Phaleria macrocarpa (Scheff.) Boerl Extract on Malondialdehyde (MDA) Level in Preeclampsia-Induced Human Umbilical Vein Endothelial Cell (HUVEC) Culture
}

\author{
Leo Simanjuntak ${ }^{1,2}$, M Fidel Ganis Siregar ${ }^{3}$, Johannes C Mose ${ }^{4}$ and Sarma N Lumbanraja ${ }^{3}$ \\ ${ }^{1}$ Doctoral Program, Faculty of Medicine, Universitas Sumatera Utara, Medan, Indonesia \\ ${ }^{2}$ Department of Obstetrics and Gynecology, Faculty of Medicine, Nommensen HKBP University, Medan, Indonesia \\ ${ }^{3}$ Department of Obstetrics and Gynecology, Faculty of Medicine, Universitas Sumatera Utara, Medan, Indonesia \\ ${ }^{4}$ Department of Obstetrics and Gynecology, Faculty of Medicine, Universitas Padjadjaran, Bandung, Indonesia \\ *Corresponding Author: Leo Simanjuntak, Doctoral Program, Faculty of Medicine, Universitas Sumatera Utara and Department of \\ Obstetrics and Gynecology, Faculty of Medicine, Nommensen HKBP University, Medan, Indonesia.
}

Received: June 11, 2019; Published: July 11, 2019

DOI: $10.31080 /$ ASMS.2019.03.0346

\begin{abstract}
Preeclampsia is a major cause in both maternal and perinatal mortality and morbidity. The etiopathogenesis of preeclampsia remains unclear but endothelial dysfunction is one of the leading theories. Increased oxidative stress and lipid peroxides and reduced antioxidants play important role in the pathophysiology of preeclampsia. Malondialdehyde (MDA) is the final product of lipid peroxidation, used as the oxidative stress marker. HUVEC (Human Umbilical Vein Endothelial Cell) culture is an in vitro model widely used to study the pathogenesis of preeclampsia. Phaleria macrocarpa (Scheff.) Boerl also known as Mahkota Dewa is widely used as an anti-inflammation and antioxidant because of alkaloids, saponins, flavonoids and polyphenols properties. This study aimed to determine the effects of Phaleria macrocarpa (Scheff.) Boerl Extract on oxidative stress in endothelial cells by measuring the MDA level in preeclampsia-induced HUVEC. Our results showed the Phaleria macrocarpa's extract reduce MDA level siginificantly at concentration of $3.906 \mu \mathrm{g} / \mathrm{mL}$. Phaleria macrocarpa's extract at concentration of $15.625 \mu \mathrm{g} / \mathrm{mL}$ reduce MDA level to normal level. Thus, Phaleria macrocarpa's extract might be used as agent to overcome oxidative stress in preeclampsia.
\end{abstract}

Keywords: Phaleria macrocarpa; Preeclampsia; HUVEC; MDA; Oxidative Stress

\section{Introduction}

Preeclampsia is one of the leading causes of maternal morbidity and mortality worldwide. It is estimated that maternal deaths worldwide are around 500,000 annually and about 10\% - 15\% are due to preeclampsia and eclampsia [1]. In 2006 WHO reported that $16 \%$ of maternal deaths in developed countries due to hypertension in pregnancy, higher than due to bleeding of $13 \%$, abortion of $8 \%$ and sepsis of $2 \%$ [2].

Although there have been many studies but the etiopathogenesis of preeclampsia is still not fully elucidated but it is believed to be a multifactors. Thus preeclampsia is called the 'disease of theories' [3].

Endothelial dysfunction plays an important role in the pathophysiology of preeclampsia. Under normal circumstances, endothelial cells maintain vascular integrity, regulating blood pressure, preventing intravascular coagulation, and regulating vascular smooth muscle tone by producing various substances including nitric oxide (NO), endothelin, prostacyclin and thromboxane [4]. Endothelial dysfunction occurs due to cytotoxic factors in the circulation such as superoxide anions $\left(\mathrm{O}_{2}^{-}\right)$and $\mathrm{H}_{2} \mathrm{O}_{2}$, trophoblast debris, pro-inflammatory cytokines, metabolic factors, and anti-angiogenic factors produced by oxidative stressed placenta and causing excessive maternal inflammatory responses.

Oxidative stress is considered as a mediator of endothelial dysfunction in preeclampsia [5]. Pregnancy itself is a state of oxidative stress along with mitochondrial activity and increased production of Reactive oxygen species (ROS) but is offset by increased antioxidants production. Increased oxidative stress and lipid peroxides and reduced antioxidants play a role in the pathophysiology of preeclampsia [6-8]. Wang and Walsh [9] found there is elevated levels of lipid peroxide in preeclampsia placental tissue compared to normal pregnancy placental tissue. 
Malondialdehyde (MDA) is the final product of lipid peroxidation, thus it is used as one of the oxidative stress marker. Madazli., et al. [10] found that MDA levels in preeclampsia placental and plasma were significantly higher than normal pregnancies (6.06 \pm $0.94 \mathrm{nmol} / \mathrm{ml}$ vs $4.49 \pm 0.71 \mathrm{nmol} / \mathrm{ml})$ and $(10.18 \pm 1.32 \mathrm{nmol} / \mathrm{gr}$ wet weight vs $6.72 \pm 1.27 \mathrm{nmol} / \mathrm{gr}$ wet weight). Whereas superoxide dismutase (SOD) levels decreased significantly in preeclampsia placental and plasma than normal pregnancies $(23,51 \pm 2,27 \mathrm{U} / \mathrm{ml}$ vs $26,57 \pm 1,44 \mathrm{U} / \mathrm{ml})$ and $(40,83 \pm 9,62 \mathrm{U} /$ gr wet weight vs 50,43 $\pm 16,87 \mathrm{U} /$ gr wet weight).

Preeclampsia treatment will only be successful and rational if based on understanding the disease pathophysiology. In an attempt to determine the pathophysiology of a disease, in vitro model research is considered the best and most effective way [11]. HUVEC (Human Umbilical Vein Endothelial Cell) cell line culture and trophoblast cell line is an in vitro model widely used to study the pathogenesis of preeclampsia.

Herbs or medicinal plants have been used traditionally as alternative medicine since ancient times. Phaleria macrocarpa (Scheff.) Boerl also known as Mahkota dewa belongs to the Thymelaceae family, that originated from Papua province, is very popular in Indonesia used in the treatment of various diseases such as cancer, hemorrhoids, diabetes mellitus, hypertension, and others [12-14]. The phenol and flavonoid compounds in the extract of Phaleria macrocarpa have high antioxidant and anti-inflammatory activity $[14,15]$.

The aim of this study is to determine the effects of Phaleria macrocarpa (Scheff.) Boerl Extract on Malondialdehyde (MDA) Level In preeclampsia-induced Human Umbilical Vein Endothelial Cell (HUVEC).

\section{Materials and Method}

Serum samples used were obtained from women at $>20-42$ weeks of gestational age, which were diagnosed preeclampsia at Dr. Hasan Sadikin General Hospital. Research subjects have fulfilled inclusion and exclusion criteria.

\section{Cell culture}

HUVEC cell line ATCC CRL-1730 obtained from American Type Collection Culture. HUVEC cell line was growth into tissue culture flask $\left(25 \mathrm{~cm}^{2}\right)$ containing RPMI 1640 media, 20\% (v/v) FBS qualified (fetal bovine serum) supplementation, $10 \%$ endothelial supplement, 1\% Penicillin G - Streptomycin solution stabilized, and $1 \%$ antimycotic Fungizone Amphotericin B and 1\% gentamicin. The cells were then incubated at $37^{\circ} \mathrm{C}$ and $5 \% \mathrm{CO}_{2}$ (v/v). Culture medium is replaced every 2 - 3 days. Then cells are passaged every seven days until reach $80-90 \%$ confluence.

\section{Phaleria macrocarpa's extract}

Phaleria macrocarpa (Scheff.) Boerl was obtained from the Research Institute for Industrial Plants at Manoko, Lembang, West Java, Indonesia. The plant species was identified by the laboratory of Plant Taxonomy staff at Herbarium Bogoriense, Bogor, Indonesia.

\section{Measurement of MDA level}

As many as $6 \times 10^{5}$ cells $/ \mathrm{mL}$ induced with normal and preeclampsia serum, were placed into 60-well plate, then incubated at $37^{\circ} \mathrm{C}$ and $5 \% \mathrm{CO}_{2}(\mathrm{v} / \mathrm{v})$. Each well then was washed with $37^{\circ} \mathrm{C}$ PBS 3-4 times. Furthermore, various concentrations of Phaleria macrocarpa's extract $(0,977 ; 1,953 ; 3,906 ; 7,813 ; 15,625 ; 31,25 ; 62,5$; 125; and $250 \mu \mathrm{g} / \mathrm{mL}$ ) were added into each well, then incubated for 24 and 72 hours $37^{\circ} \mathrm{C}$ and $5 \% \mathrm{CO}_{2}$ (v/v). Each well then was washed with $37^{\circ} \mathrm{C}$ once for five minutes. Transfer the cells into centrifugation tube using $1.5 \mathrm{~mL}$ pipette. Centrifuged at $1.500 \mathrm{rpm}$ for 10 minutes at $4^{\circ} \mathrm{C}$. Use the supernatant as a sample for the ELISA method measurement, then the rest of the sample can be stored at $-80^{\circ} \mathrm{C}$.

\section{Data analysis}

Data was analyzed with repeated ANOVA (analysis of variance) test and followed by Bonferroni test as post hoc comparison test.

\section{Results}

As shown in figure 1 MDA levels in preeclampsia HUVEC culture model is higher than normal pregnancy HUVEC culture model. The MDA levels at 72 hours incubation time was lower than the 24 hours incubation time in both normal and preeclampsia models. Figure 2 shows that LC3-II levels in preeclampsia HUVEC culture model is higher than normal pregnancy HUVEC culture model. The LC3-II levels at 72 hours incubation time was higher than the 24 hours incubation time in both normal and preeclampsia models.

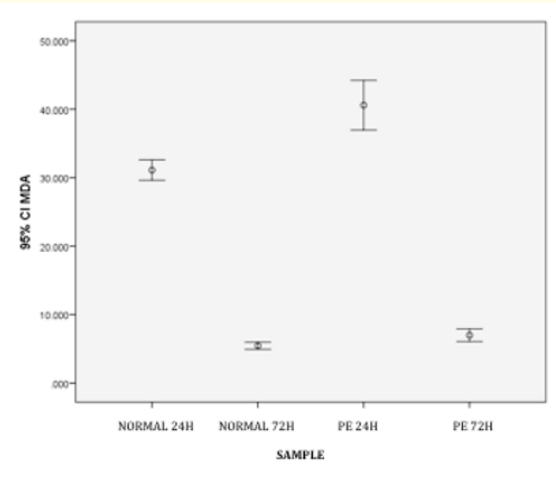

Figure 1: MDA levels in normal and preeclampsia-induced HUVEC based on incubation time. 
The Effects of Phaleria macrocarpa (Scheff.) Boerl Extract on Malondialdehyde (MDA) Level in Preeclampsia-Induced Human Umbilical Vein Endothelial Cell (HUVEC) Culture

Table 1 shows MDA levels mean in difference preeclampsia and normal serum-induced HUVEC culture model treated with Phaleria macrocarpa's extract in various concentrations incubated for 24 and 72 hours.
Table 2 shows MDA level decreased in preeclampsia seruminduced HUVEC ATCC CRL 1730 following increased Phaleria macrocarpa's extract concentration. MDA level significantly decreased after exposure of Phaleria macrocarpa's extract on concentration $3.906 \mu \mathrm{g} / \mathrm{mL} .(\mathrm{p}<0,05)$.

\begin{tabular}{|l|c|c|c|c|}
\hline \multirow{2}{*}{$\begin{array}{c}\text { Phaleria macrocarpa's extract } \\
\text { concentration }(\boldsymbol{\mu g} / \mathbf{m L})\end{array}$} & \multicolumn{2}{c|}{ 24 H INCUBATION TIME } & \multicolumn{2}{c|}{ 72 H INCUBATION TIME } \\
\cline { 2 - 5 } & NP* (Mean \pm SD) & PE (Mean \pm SD) & NP* (Mean \pm SD) & PE (Mean \pm SD) \\
\hline Control & $37.520 \pm 0.358$ & $53.978 \pm 0.028$ & $6.906 \pm 0.086$ & $10.772 \pm 0.006$ \\
\hline 0.977 & $32.951 \pm 0.068$ & $48.063 \pm 0.003$ & $5.947 \pm 0.002$ & $7.824 \pm 0.001$ \\
\hline 1.953 & $31.488 \pm 0.564$ & $45.483 \pm 0.672$ & $5.847 \pm 0.007$ & $7.219 \pm 0.001$ \\
\hline 3.906 & $31.033 \pm 0.062$ & $39.064 \pm 0.008$ & $5.450 \pm 0.001$ & $6.563 \pm 0.003$ \\
\hline 7.813 & $29.882 \pm 0.013$ & $39.545 \pm 0.001$ & $5.232 \pm 0.001$ & $6.323 \pm 0.003$ \\
\hline 15.625 & $29.004 \pm 0.003$ & $37.260 \pm 0.353$ & $5.083 \pm 0.008$ & $6.126 \pm 0.001$ \\
\hline 31.25 & $28.987 \pm 0.006$ & $35.237 \pm 0.007$ & $4.901 \pm 0.006$ & $5.913 \pm 0.003$ \\
\hline 62.5 & $28.360 \pm 0.494$ & $33.095 \pm 0.008$ & $4.611 \pm 0.077$ & $5.710 \pm 0.002$ \\
\hline 125 & $28.010 \pm 0.000$ & $31.135 \pm 0.000$ & $4.069 \pm 0.004$ & $5.508 \pm 0.001$ \\
\hline 250 & $27.889 \pm 0.018$ & $30.566 \pm 0.006$ & $3.891 \pm 0.002$ & $4.012 \pm 0.001$ \\
\hline
\end{tabular}

Table 1: MDA levels (pmol/mL) in preeclampsia and normal serum-induced HUVEC culture model treated with Phaleria macrocarpa's extract in various concentrations incubated for 24 and 72 hours.

NP: Normal Pregnancy

\begin{tabular}{|l|c|c|c|c|}
\hline \multirow{2}{*}{$\begin{array}{c}\text { Phaleria macrocarpa's extract } \\
\text { concentration }(\boldsymbol{\mu g} / \mathbf{m L})\end{array}$} & \multicolumn{2}{|c|}{ 24 H INCUBATION TIME } & \multicolumn{2}{c|}{ 72 H INCUBATION TIME } \\
\cline { 2 - 4 } & PE (Mean \pm SD) & P value* & PE (Mean \pm SD) & P value* $^{*}$ \\
\hline Control & $53.978 \pm 0.028$ & & $10.772 \pm 0.006$ & 0.034 \\
\hline 0.977 & $48.063 \pm 0.003$ & 0.085 & $7.824 \pm 0.001$ & 0.032 \\
\hline 1.953 & $45.483 \pm 0.672$ & 1.000 & $7.219 \pm 0.001$ & 0.044 \\
\hline 3.906 & $39.064 \pm 0.008$ & 0.027 & $6.563 \pm 0.003$ & 0.016 \\
\hline 7.813 & $39.545 \pm 0.001$ & 0.037 & $6.323 \pm 0.003$ & 0.022 \\
\hline 15.625 & $37.260 \pm 0.353$ & 0.009 & $6.126 \pm 0.001$ & 0.015 \\
\hline 31.25 & $35.237 \pm 0.007$ & 0.022 & $5.913 \pm 0.003$ & 0.034 \\
\hline 62.5 & $33.095 \pm 0.008$ & 0.019 & $5.710 \pm 0.002$ & 0.022 \\
\hline 125 & $31.135 \pm 0.000$ & 0.026 & $5.508 \pm 0.001$ & 0.017 \\
\hline 250 & $30.566 \pm 0.006$ & 0.019 & $4.012 \pm 0.001$ & \\
\hline
\end{tabular}

Table 2. MDA levels (pmol/mL) mean comparison before and after various concentrations of Phaleria macrocarpa's extract treatment at 24 hours and 72 hours incubation time in preeclampsia HUVEC culture model.

*: statistically significant if $\mathrm{p}<0.05$

Figure 2 shows that Phaleria macrocarpa's extract at concentration of $15.625 \mu \mathrm{g} / \mathrm{mL}$ reduce MDA level in preeclampsia model to normal pregnancy level.

\section{Discussion}

This were the first study to evaluate the effects of Phaleria macrocarpa (Scheff.) Boerl extract on Malondialdehyde (MDA) level in Preeclampsia-Induced Human Umbilical Vein Endothelial Cell (HU- 
The Effects of Phaleria macrocarpa (Scheff.) Boerl Extract on Malondialdehyde (MDA) Level in Preeclampsia-Induced Human Umbilical Vein Endothelial Cell (HUVEC) Culture

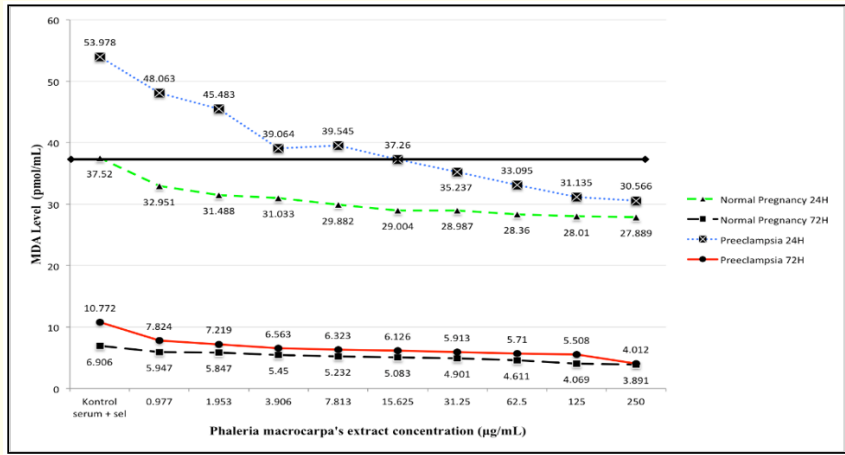

Figure 2: MDA levels in relation with Phaleria macrocarpa's extract concentration

VEC). Preeclampsia and eclampsia have been known since ancient times but their pathophysiology is still not clearly understood.

There is compelling evidence that endothelial dysfunction plays a role in the pathophysiology of preeclampsia. A consistent finding is the presence of glomerular endotheliosis in more than $70 \%$ of primiparous preeclampsia patients and this glomerular endotheliosis will disappear after delivery.

To date, invitro research using HUVEC has been done a lot recently. Previous invitro research on HUVEC cultures by treating with anti-inflammatory and antioxidant compounds such as curcumin and Papua ant nest (Myrmecodia pendens) decrease oxidative stress and inflammation characterized by decreased levels of MDA, and TNF- $\alpha$. These studies conclude that the Papuan ant nests and curcumin have a therapeutic effect on preeclampsia $[16,17]$.

Oxidative stress is considered as a mediator of endothelial dysfunction in preeclampsia [5]. Malondialdehyde (MDA) is the final product of lipid peroxidation, thus it is used as one of the oxidative stress marker. Autophagy can be induced by many overlapping factors such as nutritional deficiencies, growth factors deficiencies, and intracellular stress due to hypoxia. LC3-II is used as a typical marker of autophagosome formation in autophagy.

In this study results showed MDA levels in preeclampsia HUVEC culture model was higher than normal pregnancy HUVEC culture model. MDA level decreased in preeclampsia and normal seruminduced HUVEC ATCC CRL 1730 culture following increased Phaleria macrocarpa's extract concentration. MDA level significantly decreased after exposure of Phaleria macrocarpa's extract on con- centration $3.906 \mu \mathrm{g} / \mathrm{mL}$. Phaleria macrocarpa's extract at concentration of $15.625 \mu \mathrm{g} / \mathrm{mL}$ reduce MDA level to normal level.

The result of present study suggests that Phaleria macrocarpa's extract contains anti-oxidant activity proven by decreased level of MDA. It was also described that MDA level decreased in preeclampsia and normal serum-induced HUVEC ATCC CRL 1730 following increased Phaleria macrocarpa's extract concentration. Thus, Phaleria macrocarpa's extract might be used as agent to overcome oxidative stress in preeclampsia. Since the decreased the level of MDA in preeclampsia-induced HUVEC ATCC CRL 1730 culture, further clinical studies regarding the use of Phaleria macrocarpa's extract in treatment are encouraged.

\section{Conclusion}

The Phaleria macrocarpa's extract reduce MDA level siginificantly at concentration of $3.906 \mu \mathrm{g} / \mathrm{mL}$ in preeclampsia-induced HUVEC ATCC CRL 1730 culture. Phaleria macrocarpa's extract at concentration of $15.625 \mu \mathrm{g} / \mathrm{mL}$ reduce MDA level to normal level.

\section{Bibliography}

1. Maynard S., et al. "Preeclampsia and Angiogenic Imbalance". Annual Review of Medicine 59 (2008): 61-78.

2. Cunningham FG., et al. Williams Obstetrics 24th. ed. McGraw Hill Medical (2014): 728-779.

3. Raghupathy R. "Cytokines as a Key Players in the Pathophysiology of Preeclampsia". Medical Principles and Practice 22.1 (2013): 8-19.

4. George EM and Granger JP. "Endothelin : Key Mediator of Hypertension in Preeclampsia". American Journal of Hypertension 24.9 (2011): 964-969.

5. Davidge ST., et al. "Endothelial Cell Dysfunction, in Taylor, RN, Roberts, JM, Cunningham, FG and Lindheimer, MD, Eds, Chesley's Hypertensive Disorders in Pregnancy Fourth Edition, Elsevier Inc (2015): 181-207.

6. Wang Y and Walsh SW. "TNF- $\alpha$ concentration and mRNA expression are increased in preeclamptic placenta". American Journal of Reproductive Immunology 32 (1996): 157-169.

7. Walsh SW. "The Role of oxidative stress and antioxidants in preeclampsia". Contemporary Obstetrics and Gynecology 42 (1997): 113-124. 
8. Redman CWG and Sargent IL. "Placental Debris, Oxidative Stress and Pre-eclampsia”. Placenta 21 (2000): 597-602.

9. Wang GL., et al. "Hypoxia-inducible factor 1 is a basic-helixloop-helix-PAS heterodimer regulated by cellular 02 tension". Proceedings of the National Academy of Sciences of the United States of America 92 (1995): 5510-5514.

10. Madazli R., et al. "The plasma and placenta levels of malondialdehyde, gluthation and superoxide dismutase in preeclampsia”. Journal of Obstetrics and Gynecology 22.5 (2002): 477-480.

11. Orendi K., et al. "Placental and trophoblastic in vitro models to study preventive and therapeutic agents for preeclampsia". Placenta 32 (2011): 549-554

12. Anggraini T and Lewandowsky P. "The exotic plants of Indonesia: Mahkota Dewa (Phaleria macrocarpa), Sikaduduak (Melastoma malabathricum Linn) and Mengkudu (Morinda citrifolia) as potent antioxidant sources". International Journal of Advanced Science and Engineering 5 (2015): 59-62.

13. Alara OR., et al. "Review on Phaleria macrocarpa Pharmacological and Phytochemical Properties". Drug Designing, Open Access (2016).

14. Hendra R., et al. "Antioxidant, Anti-inflammatory and Cytotoxicity of Phaleria Macrocarpa (Boerl.) Scheff Fruit". BMC Complementary and Alternative Medicine 11 (2011): 110.

15. Tiwari P., et al. "Phytochemical screening and extraction: a review". Internationale Pharmaceutica Sciencia 1.1 (2011): 98-106.

16. Yeni CM., et al. "Effect of Curcumin in Decreasing MDA Level in Preeclampsia-induced Human Umbilical Vein Endothelial Cells (HUVEC)". International Journal of Pharm Tech Research 10.2 (2017): 69-72.

17. Gunardi JI., et al. "Effects of Papua Ant Nests (Myrmecodia pendens) on Level of sFlt-2, PIGF, MDA, and NO in Preeclampsia-induced HUVEC Cell Line". International Journal of Pharm Tech Research 6 (2016): 424-435.

\section{Volume 3 Issue 8 August 2019}

(C) All rights are reserved by Leo Simanjuntak, et al. 